\title{
Is the future of symptomatic intracranial atherosclerotic stenosis management promising?
}

\author{
Dacheng Liu, ${ }^{1}$ Jingyi Liu, ${ }^{1}$ Yuan Cai, ${ }^{1}$ Ka Sing Lawrence Wong, ${ }^{2}$ \\ Liping Liu $\circledast^{1,3}$
}

Intracranial atherosclerotic stenosis (ICAS) is one of most common causes of stroke; it has the highest rate of recurrence compared with other aetiologies. ${ }^{1}$ ICAS is especially more prevalent among the Asians. ${ }^{2}$ The results of the Chinese Intracranial Atherosclerosis Study indicated that the prevalence of symptomatic ICAS (sICAS) is as high as $46 \% .^{1}$ The 1 -year stroke recurrence rate with sICAS is reported to be $12.2 \%$ and $15 \% .^{34}$ We understand, the following pathological reasons could account for the high recurrence rate in sICAS. First, patients with ICAS are prone to encounter hypoperfusion haemodynamics, especially under poor collateral conditions. ${ }^{5}$ Studies on the association of blood pressure (BP) control in the acute stage and clinical outcomes are contradictory, supporting this hypothesis. ${ }^{6}$ Second, plaques in stenotic arteries are usually highly vulnerable (due to large lipid cores, thin fibrous caps and intraplaque haemorrhage). ${ }^{7}$ These plaques are prone to rupture into downstream arteries, leading to embolic stroke in the territory of the responsible artery. ${ }^{5}$ Furthermore, these patients are often comorbid with heightened risk factors strongly correlated with stroke recurrence, including hypertension, metabolic disorders, dyslipidaemia. ${ }^{8}$

Therefore, there is an urgent need to standardise assessment and management of sICAS. The treatment of sICAS nowadays primarily focuses on secondary prevention to reduce stroke recurrence. Therapeutic methods include antiplatelet therapy, interventional therapy and control of risk factors. ${ }^{9}$ Two milestone trials, the Warfarin Aspirin Symptomatic Intracranial Disease (WASID) ${ }^{3}$ Trial

\footnotetext{
${ }^{1}$ Department of Neurology, Beijing Tiantan Hospital, Capital Medical University, Beijing, China

${ }^{2}$ Division of Neurology, Department of Medicine and Therapeutics, Chinese University of Hong Kong, Prince of Wales Hospital, Hong Kong, China

${ }^{3}$ China National Clinical Research Center for Neurological Diseases, Beijing, China
}

Correspondence to Dr Liping Liu, Department of Neurology, Beijing Tiantan Hospital, Capital Medical University, Beijing 100070, China; lipingsister@gmail. com and the Stenting and Aggressive Medical Management for Preventing Recurrent Stroke in Intracranial Stenosis (SAMMPRIS) ${ }^{4}$ Trial aim to provide an updated strategy for clinical practice.

The WASID Trial, with a double-blind, multicentre, randomised control clinical trial design, aimed to evaluate the efficacy and safety of warfarin over aspirin among patients with 50\%-99\% degree of ICAS. Aspirin proved to be equally efficient and safer. This led to the investigation of a dual-antiplatelet therapy for secondary prevention in patients with sICAS. The Clopidogrel Plus Aspirin versus Aspirin Alone for Reducing Embolization in Patients with Acute Symptomatic Cerebral or Carotid Artery Stenosis ${ }^{10}$ Trial indicated that in patients in the sICAS group, dualantiplatelet therapy reduced microembolic signals detected by transcranial Doppler ultrasound (TCD) (31\% vs 54\%, relative risk reduction $42.4 \%, 95 \% \mathrm{CI} 4.6 \%$ to $65.2 \%, p=0.025)$ at day 2 of enrolment, which may further reduce the recurrence of artery-origin embolic stroke in these patients. The Clopidogrel in High-Risk Patients with Acute Non-disabling Cerebrovascular Events ${ }^{11}$ Trial published in 2013 first verified the benefit of dualantiplatelet therapy for minor strokes and high-risk transient ischaemic attacks. It showed that combined use of clopidogrel and aspirin started within 24 hours and continued to 21 days was superior to aspirin monotherapy in reducing the risk of stroke in the first 90 days. In the ICAS subgroup analysis, ${ }^{12}$ it was found that dual-antiplatelet therapy had a tendency to reduce stroke recurrence over monoantiplatelet therapy at 90 days, but was not statistically significant due to the limited number of participants enrolled.

Interventional therapy beyond the conventional reperfusion therapeutic time window in acute ischaemic stroke (AIS) is considered to modify stenotic status, improve haemodynamics and further reduce stroke recurrence, but its efficacy remains controversial. In the SAMMPRIS Trial, Chimowitz and colleagues ${ }^{4}$ attempted to observe whether angioplasty or stenting plus medical therapy is beneficial for patients with sICAS over medical therapy alone. 451 patients with sICAS of 70\%-99\% stenosis within 30 days of onset enrolled. During the 30-day follow-up, $14.7 \%$ in the stenting group reached primary end points of stroke recurrence or death, versus only $5.8 \%$ in the medical management group $(p=0.002)$. Interestingly, the authors further analysed the outcome for those patients who met the SAMMPRIS criteria from the WASID Trial: ${ }^{13}$ the 30 -day and 1 -year stroke recurrence and death rates in the WASID Trial are much higher than those in the SAMMPRIS Trial (10.5\% vs 5.8\%, $21.9 \%$ vs $12.6 \%$, respectively, $\mathrm{p}=0.009$ ). Interpreting the results, the authors found that aggressive control of risk factors and the ideal target in the SAMMPRIS Trial medical group may contribute to the difference. Therefore, aggressive medical treatment is still the main therapeutic strategy for secondary prevention of sICAS.

Despite the emerging research results, there still persist lots of problems and concerns in clinical practice. Risk factor management targets have not yet been verified. Most of the risk factors of sICAS are managed according to SAMPPRIS standards: systolic blood pressure (SBP) $\leq 140 \mathrm{~mm} \mathrm{Hg}(\leq 130 \mathrm{~mm} \mathrm{Hg}$ if diabetes), low-density lipoprotein cholesterol (LDL-C) level reduced by $50 \%$ or $<1.8 \mathrm{mmol} / \mathrm{L}$. Increased SBP is strongly associated with a high recurrence rate in ICAS. ${ }^{14}$ However, for patients with AIS with large intracranial vessel stenosis, and potential for hypoperfusion disorders, the ideal BP target and treatment initiation timing remains unclear. It is reasonable to assume that many physicians are cautious about antihypertension treatment in patients with ICAS, especially during the acute stage.

As for dyslipidaemia, the Stroke Prevention by Aggressive Reduction in Cholesterol Levels (SPARCL) ${ }^{15}$ Trial provided evident reduction in the rate of stroke recurrence with intensive statin therapy. But since SPARCL did not specially focus on patients with ICAS, this result should be interpreted with caution. The significance of statin therapy in reducing stroke recurrence in ICAS has only been reported in observational studies. ${ }^{16}$

Moreover, despite aggressive medical treatment, some patients with sICAS still face a high stroke recurrence rate. Many researchers have attempted to improve current medical treatment strategy. For example, resorting to new antiplatelet agents such as cilostazol ${ }^{17}$ 
and ticagrelor. ${ }^{18}$ But the efficacy of these antiplatelet medicines hasn't been established yet. Besides, some patients with ICAS fail to have satisfactory lowering of LDL-C with intensive statin therapy. Thus, proprotein convertase subtilisinkexin type 9 inhibitors, such as alirocumab, ${ }^{19}$ which could lower LDL-C levels significantly, could be the alternative choice. But their application in ICAS treatment still has a long way to go.

In addition, the role of surgical methods, such as intervention in sICAS, remains uncertain. Reperfusion therapy has been the first-line treatment for AIS with large artery occlusion within the therapeutic time window, from recent successful randomised controlled trials (RCTs). ${ }^{20}$ But for sICAS at the subacute stage, the SAMMPRIS Trial and the Vitesse Intracranial Stent Study for Ischaemic Stroke Therapy ${ }^{21}$ Trial failed to prove the importance of priority of stenting over aggressive medical treatment. Some concerns emerged regarding the results of these two trials: participants were selected only through severe stenosis, regardless of their perfusion or collateral circulation status; the relative limited experience of the subcentres; the second-line devices used in these trials may not be ideal for all complex vascular situations. In a recent Chinese sICAS Registry (NCT01968122), ${ }^{22}$ all participants were enrolled for hypoperfusion aetiology with poor collaterals within 90 days after onset, and received individualised endovascular treatment. Patients had $4.3 \%$ and $8.1 \%$ of stroke recurrence at 30 days and 1 year, respectively, ${ }^{22}$ which is quite lower than in the SAMMPRIS Trial. Despite ethnic differences between various trials, we believe that the results of the Chinese Trial were better mainly due to the highly selective patient enrolment. Future RCTs comparing individually tailored stenting versus medical therapy in a specially selected cohort of patients are particularly needed. Another surgical alternative that should be considered is extracranial-intracranial (EC-IC) bypass surgery. In a recent cohort study, ${ }^{24}$ the superficial temporal artery-middle cerebral artery bypass in carefully selected patients with severe intracranial stenoocclusive disease could improve cerebral haemodynamic parameters, which further prevent recurrence of ischaemic stroke during a follow-up of 34 months. However, there is a lack of high-level evidence of an RCT on individual assessment, and the indication and efficacy of EC-IC bypass is still controversial.
Lastly, the process of recognition and assessment of features of ICAS are still under progress. Commonly used methods for vascular and perfusion assessments include TCD, MR angiography, arterial spin labelling, CT angiography, CT perfusion, digital subtraction angiography. Compared with the above imaging methods, high-resolution MRI (HR-MRI), which could provide additional valuable information on the diagnosis of ICAS and plaque, has been further studied and is widely used. HR-MRI could particularly detect plaque components, vessel wall morphology and luminal thrombus. ${ }^{7}$ Clinical researchers have considered variation of plaque characteristics on HR-MRI as the primary end points, for further use in clinical practice. Trials detecting the effect of medical therapy guided by HR-MRI in ICAS should be one of the directions in future $^{25}$. Furthermore, recent research on the computational fluid dynamics $(\mathrm{CFD})^{26}$ technique providing noninvasive utilisation of dynamic features such as translesional pressure ratio and translesional wall shear stress ratio and clinical relevance of patients with ICAS, might be a promising and helpful guide to a better individualised secondary prevention strategy.

In conclusion, sICAS has been one of the top burdens of stroke, worldwide. Antiplatelet therapy, interventional therapy and control of critical risk factors are the major therapeutic approaches on hand. To further reduce the recurrence of stroke and improve outcome, the potential management strategy could be alternative drugs for antiplatelet and lipid lowering, more precise targets for control of risk factors, and validating particular patients benefiting from interventional therapy. New imaging techniques, such as HR-MRI and CFD, may provide detailed understanding of the pathology and mechanism, significant for governing the risk factors, and reducing risk of recurrent stroke in sICAS. In future, larger-scale studies with more specific patient data are warranted for individualised secondary prevention.

Contributors DL, JL and YC collected data and drafted the manuscript, KW revised the manuscript. LL designed and revised the manuscript.

Funding This work was supported by the National Key R\&D programme of China (2016YFC1307301) and National Natural Science Foundation of China (81820108012).

Competing interests None declared.

Patient consent for publication Not required.
Provenance and peer review Commissioned; externally peer reviewed.

\section{0 OPEN ACCESS}

Open access This is an open access article distributed in accordance with the Creative Commons Attribution Non Commercial (CC BY-NC 4.0) license, which permits others to distribute, remix, adapt, build upon this work non-commercially, and license their derivative works on different terms, provided the original work is properly cited, appropriate credit is given, any changes made indicated, and the use is non-commercial. See: http://creativecommons.org/ licenses/by-nc/4.01.

(C) Author(s) (or their employer(s)) 2020. Re-use permitted under CC BY-NC. No commercial re-use. See rights and permissions. Published by BMJ.

\section{(A) Check for updates}

To cite Liu D, Liu J, Cai Y, et al. J Neurol Neurosurg Psychiatry 2020;91:122-124.

Received 8 August 2019

Revised 18 November 2019

Accepted 26 November 2019

Published Online First 16 December 2019

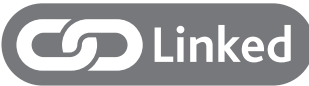

- http://dx.doi.org/10.1136/jnnp-2019-320893

J Neurol Neurosurg Psychiatry 2020;91:122-124. doi:10.1136/jnnp-2019-321564

ORCID iD

Liping Liu http://orcid.org/0000-0003-2943-055X

\section{REFERENCES}

1 Wang Y, Zhao X, Liu L, et al. Prevalence and outcomes of symptomatic intracranial large artery stenoses and occlusions in China: the Chinese intracranial atherosclerosis (CICAS) study. Stroke 2014;45:663-9.

2 Holmstedt CA, Turan TN, Chimowitz MI. Atherosclerotic intracranial arterial stenosis: risk factors, diagnosis, and treatment. Lancet Neurol 2013;12:1106-14.

3 Chimowitz MI, Lynn MJ, Howlett-Smith $\mathrm{H}$, et al. Comparison of warfarin and aspirin for symptomatic intracranial arterial stenosis. N Engl J Med 2005;352:1305-16.

4 Chimowitz MI, Lynn MJ, Derdeyn CP, et al. Stenting versus aggressive medical therapy for intracranial arterial stenosis. N Engl J Med 2011;365:993-1003.

5 Gao S, Wang YJ, Xu AD, et al. Chinese ischemic stroke subclassification. Front Neurol 2011;2:6.

6 Hong L, Cheng X, Lin L, et al. The blood pressure paradox in acute ischemic stroke. Ann Neurol 2019:85:331-9.

7 Xu W. High-Resolution MRI of intracranial large artery diseases: how to use it in clinical practice? Stroke Vasc Neurol 2019;4:102-4.

8 Waters MF, Hoh BL, Lynn MJ, et al. Factors associated with recurrent ischemic stroke in the medical group of the SAMMPRIS trial. JAMA Neurol 2016:73:308-15.

9 Kim JS, Bang OY. Medical treatment of intracranial atherosclerosis: an update. J Stroke 2017:19:261-70.

10 Wong KSL, Chen C, Fu J, et al. Clopidogrel plus aspirin versus aspirin alone for reducing embolisation in patients with acute symptomatic cerebral or carotid artery stenosis (clair study): a randomised, open-label, blinded-endpoint trial. Lancet Neurol 2010;9:489-97. 
11 Wang Y, Wang Y, Zhao X, et al. Clopidogrel with aspirin in acute minor stroke or transient ischemic attack. $N$ Engl J Med 2013;369:11-19.

12 Liu L, Wong KSL, Leng X, et al. Dual antiplatelet therapy in stroke and ICAS: subgroup analysis of chance. Neurology 2015;85:1154-62.

13 Chaturvedi S, Turan TN, Lynn MJ, et al. Do patient characteristics explain the differences in outcome between medically treated patients in SAMMPRIS and WASID? Stroke 2015:46:2562-7.

14 Yu D-D, Pu Y-H, Pan Y-S, et al. High blood pressure increases the risk of poor outcome at discharge and 12-month follow-up in patients with symptomatic intracranial large artery stenosis and occlusions: subgroup analysis of the CICAS study. CNS Neurosci Ther 2015;21:530-5.

15 Amarenco P, Bogousslavsky J, Callahan A, et al. HighDose atorvastatin after stroke or transient ischemic attack. N Eng/ J Med 2006;355:1374-59.

16 Miao H, Yang Y, Wang H, et al. Intensive lipidlowering therapy ameliorates asymptomatic intracranial atherosclerosis. Aging Dis 2019;10:258-66.
17 Kwon SU, Hong K-S, Kang D-W, et al. Efficacy and safety of combination antiplatelet therapies in patients with symptomatic intracranial atherosclerotic stenosis. Stroke 2011:42:2883-90.

18 Amarenco P, Albers GW, Denison H, et al. Efficacy and safety of ticagrelor versus aspirin in acute stroke or transient ischaemic attack of atherosclerotic origin: a subgroup analysis of SOCRATES, a randomised, double-blind, controlled trial. Lancet Neurol 2017:16:301-10.

19 Schwartz GG, Steg PG, Szarek M, et al. Alirocumab and cardiovascular outcomes after acute coronary syndrome. N Engl J Med 2018;379:2097-107.

20 Powers WJ, Rabinstein AA, Ackerson T, et al. 2018 guidelines for the early management of patients with acute ischemic stroke: a guideline for healthcare professionals from the American heart Association/ American stroke association. Stroke 2018;49:e46-110.

21 Zaidat 00, Fitzsimmons B-F, Woodward BK, et al Effect of a balloon-expandable intracranial stent vs medical therapy on risk of stroke in patients with symptomatic intracranial stenosis: the VISSIT randomized clinical trial. JAMA 2015;313:1240-8.
22 Miao Z, Zhang Y, Shuai J, et al. Thirty-Day outcome of a multicenter registry study of stenting for symptomatic intracranial artery stenosis in China. Stroke 2015:46:2822-9.

23 Ma N, Zhang Y, Shuai J, et al. Stenting for symptomatic intracranial arterial stenosis in China: 1-year outcome of a multicentre registry study. Stroke Vasc Neurol 2018:3:176-84.

24 Low SW, Teo K, Lwin S, et al. Improvement in cerebral hemodynamic parameters and outcomes after superficial temporal artery-middle cerebral artery bypass in patients with severe stenoocclusive disease of the intracranial internal carotid or middle cerebral arteries. J Neurosurg 2015;123:662-9.

25 Chung J-W, Cha J, Lee MJ, et al. Intensive statin treatment in acute ischaemic stroke patients with intracranial atherosclerosis: a high-resolution magnetic resonance imaging study (STAMINAMRI study). J Neurol Neurosurg Psychiatry 2020;91:204-11.

26 Leng $X$, Lan L, Ip HL, et al. Hemodynamics and stroke risk in intracranial atherosclerotic disease. Ann Neurol 2019;85:752-64 